Reprod. Nutr. Dévelop., 1986, 26 (1 B), 353-354.

\title{
Effets de dérivés d'acides gras sur les ingestions volontaires d'aliments chez le mouton
}

\author{
R. PAQUAY, Françoise VANDERMEERSCHEN-DOIZÉ, Fr. VERNAILLEN
}

Laboratoire de Physiologie animale, Facultés Universitaires Notre-Dame de la Paix, B-5000 Namur, Belgique.

Summary. The continuous infusion of different fatty acid derivates (methyl, ethyl, isopropyl and ethylhexyle oleate, isopropyl myristate and ethylhexyle laurate) has the same decreasing effect on the voluntary dry matter intake of adult sheep than the infusion of oleic, palmitic and stearic acid.

Diverses expériences dans le laboratoire (Vandermeerschen-Doizé, et al., 1983 ; 1984 ; Vandermeerschen-Doizé et Paquay, 1984) ont montré que les acides gras libres du sang et plus particulièrement les acides palmitique, stéarique et oléique ont une influence sur le contrôle à long terme des ingestions volontaires d'aliments chez le mouton.

Une augmentation de la concentration sanguine de ces acides provoque une nette diminution des ingestions de matière sèche.

Les résultats de ces expériences permettent de penser qu'une réduction de la lipolyse, une diminution du transport sanguin des acides gras ou une compétition au niveau des sites d'action des acides gras pourraient modifier le comportement alimentaire des animaux. Nous avons pensé que des dérivés des acides gras pourraient avoir de telles propriétés.

Dans le but de tester cette hypothèse, divers dérivés d'acides gras (oléates de méthyle, d'éthyle, d'isopropyle et d'éthylhexyle, myristate d'isopropyle et laurate d'éthylhexyle) ont été infusés en continu à des brebis Suffolk adultes, selon un plan expérimental toujours identique.

Des groupes de 4 ou 6 brebis reçoivent à volonté du foin de prairie et des aliments composés en pellets faits de pulpes séchées de betteraves sucrières $(40 \%)$, de luzerne déshydratée $(30 \%)$ et de céréales $(30 \%)$. Les oléates de méthyle et d'éthyle d'une part, les oléates d'isopropyle et d'éthylhexyle d'autre part sont testés simultanément sur des groupes de 6 brebis divisés en 3 sousgroupes de 2. Les essais comprennent 3 périodes expérimentales de 4 jours au cours desquelles les sous-groupes reçoivent, en alternance, des infusions continues (1 1/animal/jour) d'eau physiologique (groupe témoin) ou d'eau physiologique enrichie d'un complexe albuminique des dérivés à étudier. Les périodes d'infusion sont précédées, séparées et suivies de périodes de 3 jours pendant lesquelles il n'y a pas d'infusion. Le myristate d'isopropyle et le laurate d'éthylhexyle sont testés seuls sur des groupes de 4 animaux divisés en 2 sousgroupes de 2, infusés également en alternance, pendant 4 jours d'eau physiologique et d'eau enrichie de la substance. 
Le tableau 1 donne les résultats obtenus lors de l'infusion des 6 dérivés. Chez les animaux témoins, l'infusion d'eau physiologique entraîne généralement une diminution des ingestions. Excepté pour le laurate d'éthylhexyle, cette diminution est nettement plus marquée chez les animaux expérimentaux.

\begin{tabular}{|c|c|c|c|c|c|c|c|}
\hline \multirow{3}{*}{$\begin{array}{c}\text { Dérivés } \\
\text { d'acides } \\
\text { gras } \\
\text { in f u s é s }\end{array}$} & \multirow{3}{*}{$\begin{array}{l}\text { Concen- } \\
\text { tration } \\
\text { du dérivé } \\
\text { (p. } 1000)\end{array}$} & \multicolumn{6}{|c|}{ Quantité moyenne de matière sèche ingérée en grammes par jour } \\
\hline & & \multicolumn{3}{|c|}{ Moutons expérimentaux } & \multicolumn{3}{|c|}{ Moutons témoins } \\
\hline & & $\begin{array}{c}\text { Avant } \\
F\left({ }^{*}\right) C\left(^{*}\right)\end{array}$ & $\begin{array}{l}\text { Pendant } \\
\text { F C }\end{array}$ & F $\stackrel{\text { Après }}{\mathrm{C}}$ & $\mathrm{F}^{\text {Avant }} \mathrm{C}$ & $\begin{array}{l}\text { Pendant } \\
\text { F C }\end{array}$ & F $\stackrel{\text { Après }}{\mathrm{C}}$ \\
\hline $\begin{array}{l}\text { Oléate de } \\
\text { méthyle }\end{array}$ & 5 & $\mathrm{n}^{211} 1995$ & 2191227 & 2151836 & $\begin{array}{l}205 \\
n=4\end{array}$ & 1991526 & 2151890 \\
\hline $\begin{array}{l}\text { Oléate } \\
\text { d'éthyle }\end{array}$ & 5 & $n=42010$ & 2011313 & 2171934 & $\begin{array}{l}205 \\
n=4\end{array}$ & 1991526 & 2151890 \\
\hline $\begin{array}{l}\text { Oléate } \\
\text { d'isopropyle }\end{array}$ & 5 & $n=5$ & 1661179 & 1561622 & $\begin{array}{l}178 \quad 1749 \\
n=5\end{array}$ & 1641323 & 1461771 \\
\hline $\begin{array}{l}\text { Oléate } \\
\text { d'éthylhexyle }\end{array}$ & 5 & $\mathrm{n}^{138} 1976$ & $174 \quad 788$ & 2091629 & $\begin{array}{l}178 \\
n=5\end{array}$ & 1641323 & 1461771 \\
\hline $\begin{array}{l}\text { Myristate } \\
\text { d'isopropyle }\end{array}$ & 1 & $\mathrm{n}^{212} \mathbf{1}^{1399}$ & $227 \quad 537$ & 243900 & $n^{263}=2{ }^{1737}$ & 2281386 & 2121399 \\
\hline $\begin{array}{l}\text { Laurate } \\
\text { d'éthylhexyle }\end{array}$ & 0,5 & $n_{n=2}^{184} 2139$ & 1721397 & 1601589 & $\begin{array}{l}1601589 \\
n=2\end{array}$ & 2571399 & 1841698 \\
\hline
\end{tabular}

$(*): F$ : foin : C : concentré.

Nous pouvons conclure de ces résultats que la plupart des acides gras libres ont sur le comportement alimentaire du ruminant la même action dépressive que les acides eux-mêmes.

Vandermeerschen-Doizé Fr., Bouchat J.-C., Bouckoms-Vandermeir M.-A., Paquay R., $1983 . \quad$ Effects of long-term ad libitum feeding on plasma lipid components and blood glucose, $\beta$-hydroxybutyrate and insulin concentrations in lean adult sheep. Reprod. Nutr. Déve/op., 23, 51-63.

Vandermeerschen-Doizé Fr., Paquay R., 1984. Effects of continuous long-term intravenous infusion of long-chain fatty acids on feeding behaviour and blood components of adult sheep. Appetite, 57, $137-146$.

Vandermeerschen-Doizé Fr., Bouchat J.-C., Bouckoms-Vandermeir, M.-A., Paquay R., 1984. Influence of the state of fatness on body composition and blood constituents (lipids, glucose, ketone bodies and insulin) in adult sheep. Z. Tierphysiol., Tierernährg. Futtermittelkde, 52, 105112. 